\title{
Induction of cell cycle arrest and apoptosis in myeloma cells by cepharanthine, a biscoclaurine alkaloid
}

\author{
YOSHITAKA KIKUKAWA ${ }^{1}$, YUTAKA OKUNO ${ }^{1}$, HIRO TATETSU ${ }^{1}$, \\ MIKI NAKAMURA ${ }^{1}$, NAOKO HARADA ${ }^{1}$, SHIKIKO UENO $^{1}$, \\ YORINORI KAMIZAKI $^{2}$, HIROAKI MITSUYA ${ }^{1}$ and HIROYUKI HATA ${ }^{1}$ \\ ${ }^{1}$ Department of Hematology, Kumamoto University Hospital, 1-1-1 Honjo, \\ Kumamoto 860-8556; ${ }^{2}$ Department of Otorhinolaryngology, Yatsushiro Social \\ Insurance General Hospital, 2-26 Matsuejo-machi, Yatsushiro 866-8660, Japan
}

Received May 9, 2008; Accepted July 15, 2008

DOI: 10.3892/ijo_00000068

\begin{abstract}
Cepharanthine (CEP), a biscoclaurine alkaloid extracted from Stephania Cepharantha Hayata, has been used in Japan for treating patients with radiation-induced leucopenia or thrombocytopenia. We treated a patient with multiple myeloma (MM), who was not responding to preceding chemotherapy, who coincidently received therapy with CEP due to thrombocytopenia. Since the case showed a marked reduction of tumor load, direct anti-tumor effects of CEP to myeloma cells were investigated in vitro. Anti-tumor effects were observed in all myeloma cell lines tested, including a line resistant to melphalan. Exposure to CEP of a myeloma cell line induced the production of reactive oxygen species, activated the caspase- 3 pathway and eventually induced apoptosis. Pre-exposure of cells to a pan-caspase inhibitor, Z-VAD-FMK, or a free radical scavenger, Tiron, effectively blocked CEP-induced apoptosis. Interestingly, CEP also inhibited cell growth of myeloma cells by inducing CDK inhibitors. These data show, for the first time, that CEP has anti-myeloma effects by the activation of apoptotic pathways and blocking cell cycle progression via CDK inhibitors. Although analysis of these two pathways should be clarified further, the use of CEP may be considered as a potential therapeutic agent for a subset of MM.
\end{abstract}

\section{Introduction}

The prognosis of multiple myeloma (MM) is expected to improve by the introduction of new chemotherapeutic agents such as proteasome inhibitors or thalidomide analogs in the

Correspondence to: Dr Hiroyuki Hata, Department of Hematology, Kumamoto University Hospital, 1-1-1 Honjo, Kumamoto 860-8556, Japan

E-mail: hata@kumamoto-u.ac.jp

Key words: multiple myeloma, alkaloid, CDK inhibitors, apoptosis, reactive oxygen species clinical setting $(1,2)$. However, acquisition of drug resistance is still a severe problem. Therefore, overcoming drug resistance could lead to improved survival of this disease, even in the era of new chemotherapeutic agents.

Cepharanthine (CEP) (Fig. 1) is a compound derived from Stephania Cepharantha Hayata (3) that has been widely prescribed for various diseases such as radiation-induced leucopenia (4), alopecia areata (5), exudative otitis media and venomous snakebites in Japan for $>30$ years. Since CEP is only commercially distributed in Japan, reports showing its clinical efficacy have almost been restricted to Japan. The mechanisms of this drug may be heterogeneous due to its efficacy for various diseases (6). Several investigators have reported inhibition of tumor necrosis factor (TNF)- $\alpha$-mediated $\mathrm{NF \kappa B}$ stimulation (7), perturbation of plasma membrane function to increase the intracellular accumulation of anticancer drugs (8-10), suppression of cytokine production and a protective effect against some of the disturbances mediated by proinflammatory cytokines (TNF- $\alpha$, interleukin (IL)-1ß and IL6) in septic shock (11).

Previously, CEP-induced sensitization to anticancer agents or radiation has been reported in various types of tumors (12-16). Other than combination effects, direct mechanisms for inducing cytotoxicity toward tumor cells have also been reported for CEP, such as induction of p27kip1 (17), activation of MAP kinases (18), inhibition of ATPase (19) and downregulation of AKT (20). Despite some reports showing efficacy of CEP against leukemia cells (21-23), its actions toward myeloma cells have not been reported either in vitro or in vivo. Moreover, there has been no report showing efficacy of CEP to cancer patients although a number of reports showed anti-tumor effects of CEP in vitro.

We experienced a refractory myeloma case who suffered from sudden deafness and thrombocytopenia. The case happened to be treated at the Department of Otorhinolaryngology with a combination of CEP and betamethasone because of its function in increasing platelets $(24,25)$ and improvement of sudden deafness, respectively. During the treatment of the patient, we found a marked reduction in the M-protein by this combination. This anti-myeloma effect was repeatedly observed even by monotherapy with CEP. Based on this 
finding, the mechanisms regulating the cytotoxic effects of CEP toward myeloma cells were investigated.

\section{Materials and methods}

MM cell lines and freshly purified MM cells. Five myeloma cell lines [12PE (26), U266 (27), KMM-1 (28), KHM-11 (29) and RPMI-8226 (30)] and a melphalan-resistant myeloma cell line (KHM-11 $\left.{ }^{\mathrm{EMS}}\right)(31)$ were utilized. The cells were cultured in RPMI-1640 medium and were supplemented with $10 \%$ fetal calf serum (PAA Laboratories GmbH, Linz, Austria).

Mononuclear cells were separated from bone marrow samples obtained from the patients by Ficoll-Paque Plus density sedimentation (Amersham Biosciences, Uppsala, Sweden) and subjected to CD138 immunomagnetic purification according to the protocol of the supplier (Miltenyi Biotech, Paris, France). The enriched fraction showed a purity of $>95 \%$ plasma cells as determined by May-Giemsa staining and the cell morphology. The purified tumor cells were seeded at $1 \times 10^{6}$ cells $/ \mathrm{ml}$ into round-bottomed 96-well tissue-culture plates (Falcon, Oxnard, CA), followed by the immediate addition of reagents. Informed consent was obtained from the patients.

Cell growth analysis. Cells were seeded in 96-well plates and incubated with CEP for $24 \mathrm{~h}$. The WST- 8 assay was used to evaluate the growth inhibitory effects of CEP. Briefly, $2 \times 10^{4}$ cells were incubated with various concentrations of CEP, followed by the addition of $10 \mu 1$ of WST-8 reagent (Dohjin Chemicals, Kumamoto, Japan) and incubation for $5 \mathrm{~h}$. Cell viability was determined by the light absorption at $450 \mathrm{~nm}$.

Reagents. CEP was kindly provided by Kaken Pharmaceutical (Osaka, Japan). Purified CEP and berbamine were purchased from LKT Laboratories (St. Paul, MN). Isotetrandrin was purchased from Axxora Life Sciences (San Diego, CA). Tiron (1,2-dihydroxy-3,5-benzenedisulfonic acid, disodium salt, monohydrate) was purchased from Dojindo Laboratories (Kumamoto, Japan). Tiron was dissolved in phosphatebuffered saline and used at a final concentration of $1 \mathrm{mM}$. A pan caspase inhibitor, Z-VAD-FMK (MBL, Nagoya, Japan), was dissolved in dimethyl sulfoxide and used at a final concentration of $20 \mu \mathrm{M}$.

Western blotting. Antibodies against CDK-4, CDK-6, Cyclin D1, Cyclin D3, p15-INK4B, p16-INK4A, p21Waf1/Cip1, p27Kip1, MEK and phosphorylated MEK were purchased from Cell Signaling Technology (Danvers, MA). Antibodies against caspase-3, Bax, phospho $\mathrm{Rb}$ and actin were purchased from Santa Cruz Biotechnology (Santa Cruz, CA).

Cell lysates were prepared using M-PER mammalian protein extraction reagent (Pierce Biotechnology Inc., Rockford, IL) after addition of Halt EDTA-free phosphatase inhibitor cocktail and Halt protease inhibitor cocktail (both from Pierce Biotechnology Inc.). Cell lysates were separated in NuPAGE Bis-Tris precast gels (Invitrogen, Carlsbad, CA) and transferred to PVDF membranes using an iBlot Dry Blotting system (Invitrogen). The membranes were blocked with 5\% nonfat dry milk dissolved in Tris-buffered saline (TBS; $10 \mathrm{mM}$ Tris-HCl pH 7.6, $137 \mathrm{mM} \mathrm{NaCl}$ ) containing

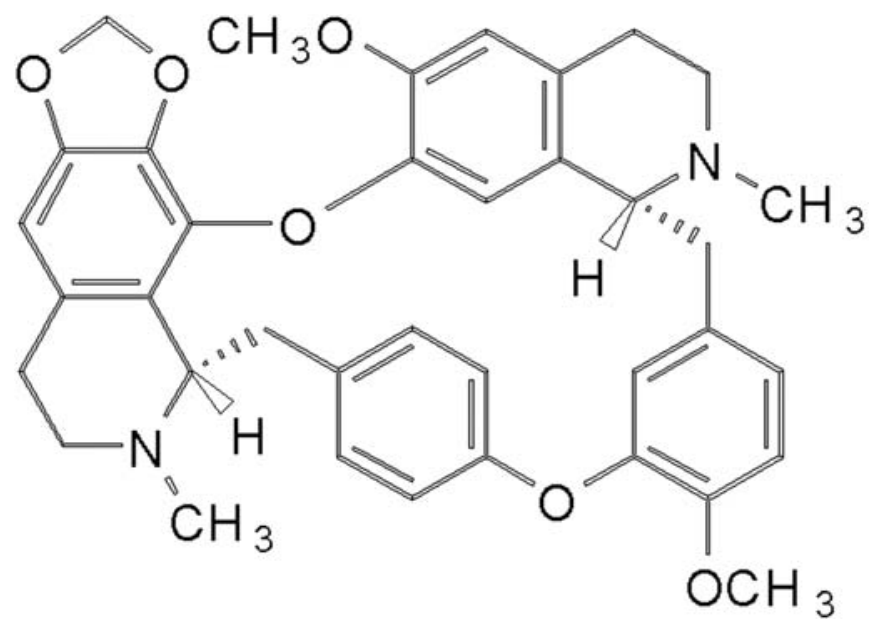

Figure 1. Chemical structures of cepharanthine.

$0.5 \%$ Tween-20 (TBS-T) for $1 \mathrm{~h}$ at room temperature, followed by incubation with a primary antibody at $4^{\circ} \mathrm{C}$ for $18 \mathrm{~h}$. For experiments detecting phosphorylated proteins, Blocking One P (Nacalai Tesque, Kyoto, Japan) was utilized to block the membranes. After three washes with TBS-T, the membranes were incubated with horseradish peroxidaseconjugated donkey anti-rabbit or sheep anti-mouse secondary antibodies (both from Amersham Biosciences, Oxford, UK) diluted in TBS-T for $2 \mathrm{~h}$ at room temperature. Antibody-bound proteins were visualized using an ECL plus kit (Amersham Biosciences) and a bio-image analyzer (Fuji Film, Tokyo, Japan).

Analysis of apoptosis. Cells were cultured for $24 \mathrm{~h}$ in the presence of reagents. Apoptosis in the MM cell lines was quantified by staining with annexin V-fluorescein isothiocyanate (FITC) and propidium iodide (PI) using an Annexin V-FITC Apoptosis Detection kit (MBL). The samples were analyzed using a flow cytometer (EPICS MCL/XL; Becton Dickinson, San Jose, CA). In some experiments, the cells were analyzed morphologically by staining with May-Giemsa solution on cytospin slides.

Evaluation of reactive oxygen species (ROS). ROS were quantified using a Live Cell Fluorescent Reactive Oxygen Species Detection kit (Marker Gene Technologies Inc., Oregon, USA) and flow cytometry. t-Butylhydroperoxide (TBHP) was utilized as a positive control.

Cell cycle analysis. The cell cycle was analyzed using a FITC-BrdU Flow kit (Becton-Dickinson and Company, Rutherford, NJ) according to the manufacturer's protocol.

\section{Results}

A case of multiple myeloma that responded to CEP. A 74-yearold female was diagnosed with MM at stage IIA in March 2001. Although she initially responded to combination chemotherapy based in MP (melphalan/prednisolone) regimen, she decided to discontinue the treatment. In 2004, MP regimen was administered again due to nasal bleeding and 
Sudden deafines:

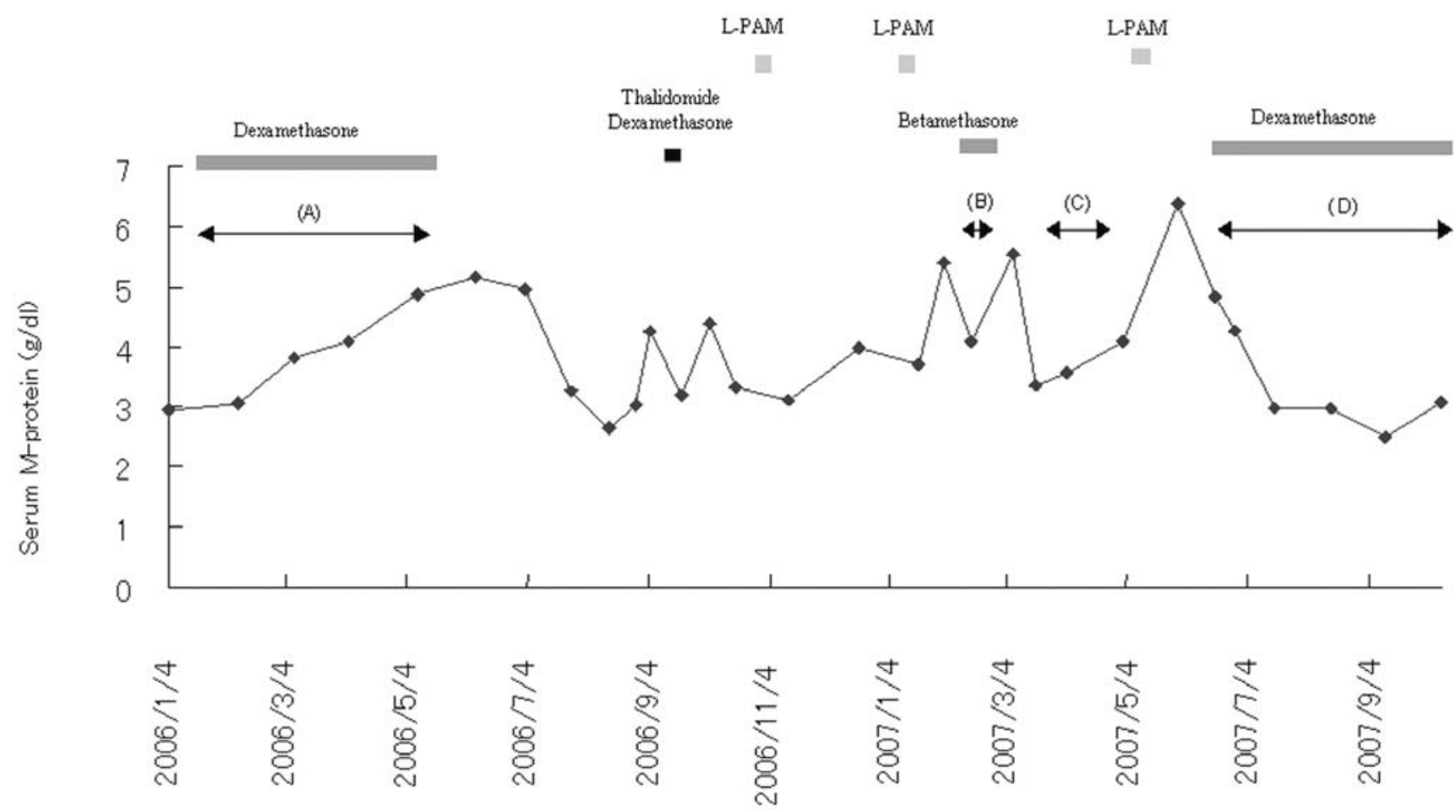

Figure 2. CEP is effective for a myeloma case. Sequential time course of M-protein is shown. A 75-year-old woman was coincidently treated with CEP and betamethasone. Although she had not been responding to dexamethasone (period A) or L-PAM, a rapid reduction in the M-protein was found during treatment with CEP and betamethasone (period B). As soon as treatment was discontinued, the M-protein increased again. A decrease of the M-protein was found by monotherapy of CEP (period C) although the response was transient. The response was observed again after combined treatment with dexamethasone and CEP (period D). It is notable that the dosage of dexamethasone utilized is the same in period A and D.

syncope, which were considered to be symptoms related to hyperviscosity. Since the response was only transient, dexamethasone was utilized at a dosage of $10 \mathrm{mg} /$ day once a week (Fig. 2, period A). However, since it was not effective, combination of thalidomide and dexamethasone was utilized. Unfortunately, thalidomide was soon discontinued due to the development of severe edema as an adverse effect of thalidomide. Since no response to dexamethasone was evident, melphalan was administered again in October 2006 and January 2007. However, as disease progressed in February 2007, platelets decreased to $25,000 / \mu 1$. At the same time, the patient suffered from sudden deafness. A combination of high dose CEP at $30 \mathrm{mg} /$ day and betamethasone starting at $3 \mathrm{mg} /$ day and declining by $0.5 \mathrm{mg} /$ day for 5 days was started by an otorhinolaryngologist in order to treat thrombocytopenia and sudden deafness, respectively.

Surprisingly, as the platelet count increased to $67,000 / \mu 1$, the concentration of the M-protein decreased from 5.4 to $4.0 \mathrm{~g} / \mathrm{dl}$ after the treatment (Fig. 2, period B). Since her Mprotein increased to $5.5 \mathrm{~g} / \mathrm{dl}$ soon after discontinuation of the treatment despite the addition of L-PAM, monotherapy of CEP started at a dosage of $30 \mathrm{mg} /$ day. Notably, the M-protein decreased again to $3.3 \mathrm{~g} / \mathrm{dl}$ by the monotherapy with CEP although the response was transient (Fig. 2, period C). Sine addition of L-PAM was not effective, dexamethasone at $10 \mathrm{mg} /$ day once a week was combined with a daily administration of
CEP. Since then, a continuous decrease in the M-protein has been observed for $>200$ days without any symptoms of hyper viscosity (Fig. 2, period D). The platelet count also improved by the treatment to the normal range. When comparing the response in period $A$ and $D$ in which the dosage of dexamethasone utilized is the same, persisted response of myeloma in period D is not considered to derive solely from dexamethasone. Taken this finding and transient response to monotherapy with CEP in period $\mathrm{C}$, further analysis was made to elucidate direct anti-myeloma effects by CEP.

Growth inhibition of myeloma cells by CEP. The human MM cell lines were cultured for $24 \mathrm{~h}$ in the presence of various concentrations of CEP and the cell viabilities were measured by the WST-8 assay. As shown in Fig. 3A, the viabilities of all the myeloma cell lines, including the melphalan-resistant myeloma cell line KHM-11 ${ }^{\mathrm{EMS}}$, were inhibited by CEP in dose-dependent manners. The $\mathrm{IC}_{50}$ values for the human $\mathrm{MM}$ cell lines ranged from 2 to $8 \mu \mathrm{M}$. $\mathrm{IC}_{50}$ of CEP to T-cell lines (MT2 and MT4) was $\sim 25 \mu \mathrm{M}$ (data not shown). Based on these results, KHM-11 and 12PE cells were further analyzed in detail as representative cell lines showing high and low sensitivity to CEP, respectively.

The morphologies of cells treated with $2 \mu \mathrm{M}$ CEP for 1,12 and $48 \mathrm{~h}$ were analyzed. Typical apoptotic features, such as fragmented nuclei with a conserved cytoplasm, were 

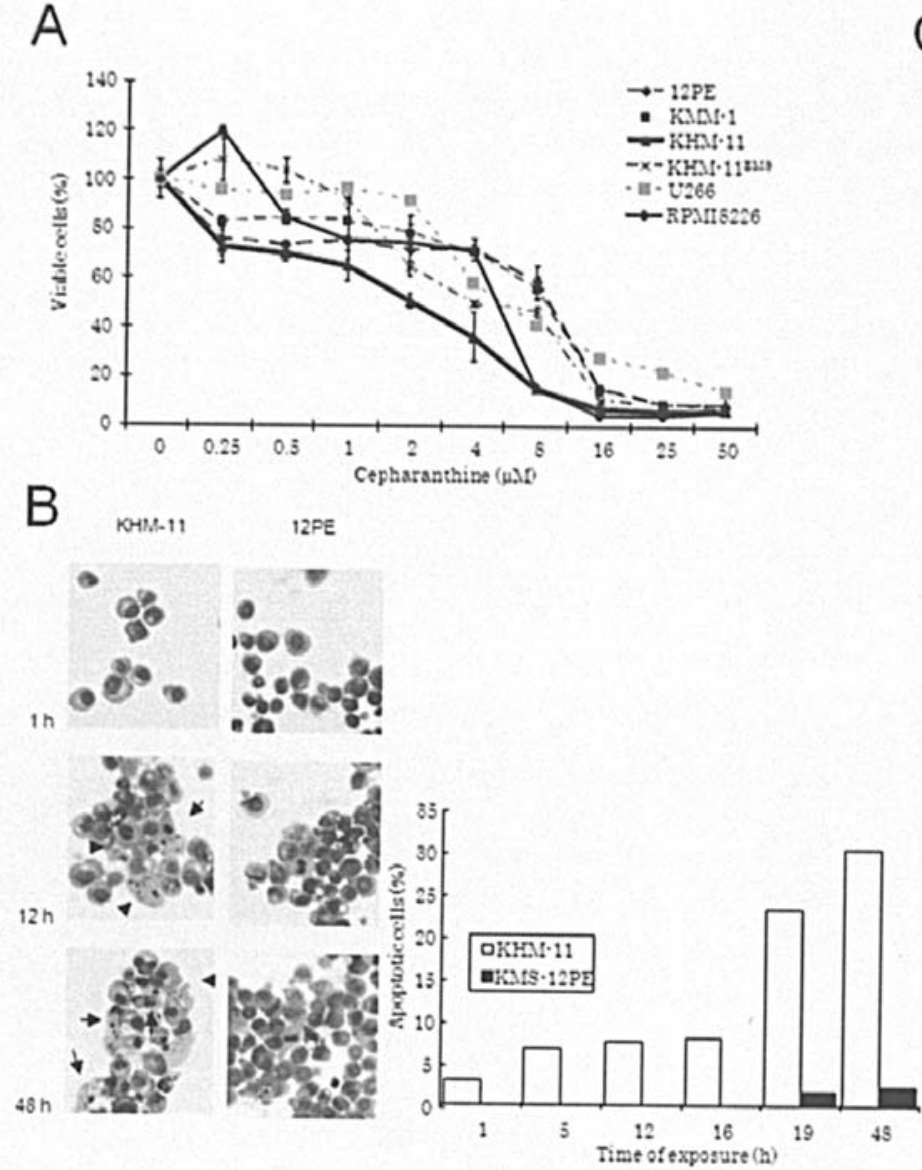

C
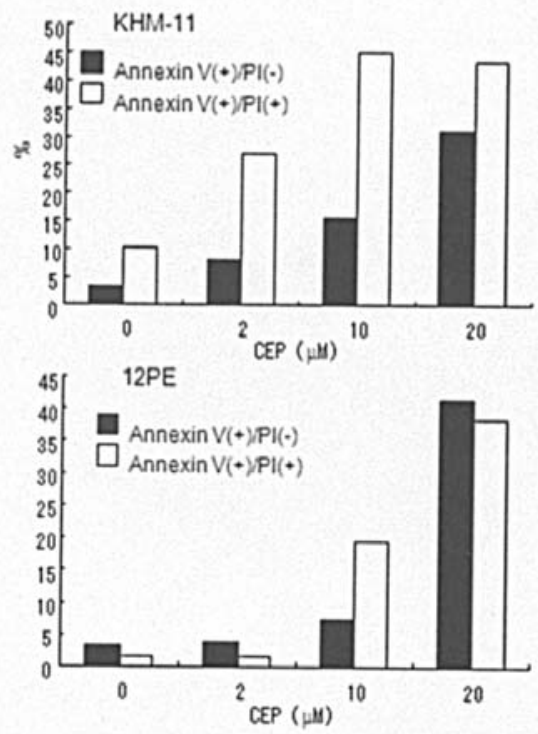

D

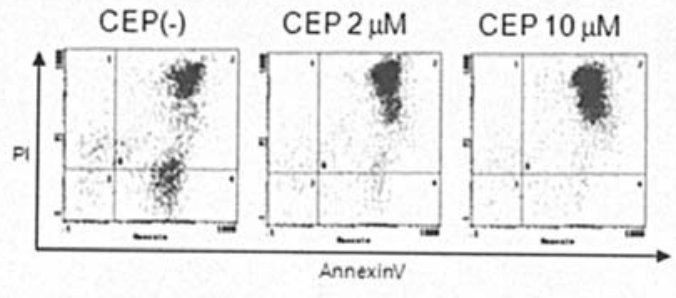

Figure 3. CEP inhibits growth of myeloma cells. (A) Six myeloma cell lines were treated with various concentrations of CEP and incubated for 24 h. Cell growth was analyzed by the WST8 assay. The percent viabilities relative to control cells are shown. All myeloma cells showed growth inhibition by CEP although their sensitivities varied. (B) CEP induces apoptosis in a time-dependent manner. Left panel, morphology of cells; right panel, quantification of apoptotic cells; open bars, KHM-11; closed bars, 12PE. KHM-11 and 12PE cells were cultured with $2 \mu \mathrm{M}$ CEP for the indicated periods. Features of apoptosis, such as a fragmented nucleus and a conserved cytoplasm, are evident in KHM-11 cells (arrows in left panel) in a time-dependent manner, but are not observed in 12PE cells. (C) Induction of apoptosis was analyzed by Annexin V/PI analysis. KHM-11 (upper panel) and 12PE (lower panel) cells were incubated with the indicated concentrations of CEP for $24 \mathrm{~h}$. Results of annexin V/PI analysis were quantified. CEP causes a marked induction of annexin V ( + ) cells in both cell lines in dose-dependent manners, although KHM-11 cells show apoptosis at a lower concentration of CEP than that in 12PE cells. (D) CEP induces apoptosis in freshly isolated CD138 (+) myeloma cells. Purified myeloma cells were incubated with CEP at the indicated concentrations for $24 \mathrm{~h}$ and analyzed by Annexin V/PI method. Although purified cells tend to undergo apoptosis under CEP (-) condition, CEP increases cell death in a dose-dependent manner.

observed in KHM-11 cells (Fig. 3B, left panel). The numbers of cells with apoptotic features were quantified and found to increase in a time-dependent manner in KHM-11 cells (Fig. 3B, right panel). However, 12PE cells showed almost no apoptosis, despite significant growth inhibition was detected by the WST-8 assay.

To further quantify the cell death, annexin V/PI analysis was performed. CEP induced annexin $\mathrm{V}$ single-positive and annexin V/PI double-positive cells in dose-dependent manners for both KHM-11 and 12PE cells (Fig. 3C). The analysis revealed that KHM-11 cells exhibited a greater susceptibility to CEP than 12PE cells, although the two cell lines showed cell death at a high concentration of CEP $(20 \mu \mathrm{M})$. When purified myeloma cells from a myeloma patient were incubated with CEP (Fig. 3D), they underwent spontaneous apoptosis under the CEP (-) condition, probably due to the absence of their microenvironment. However, the purified myeloma cells showed a marked induction of cell death by CEP, indicating that CEP accelerates cell death of primary myeloma cells in vitro.
Caspase-3 activation and ROS induction by CEP. To further characterize the mechanism of the apoptosis induced by CEP, caspase-3, Bax and reactive oxygen species (ROS) were analyzed. As shown in Fig. 4, cleaved caspase-3 (A) and increased Bax levels (B) were found in CEP-treated KHM-11 cells. However, no increase in activated caspase- 3 and no induction of Bax were observed in 12PE cells. CEP induced ROS in a dose-dependent manner in KHM-11 cells, though not in 12PE cells (Fig. 4C).

Since we detected the activation of caspase- 3 by CEP, effects of the pan-caspase inhibitor Z-VAD-FMK were analyzed (Fig. 4D). After pretreatment with Z-VAD-FMK for $30 \mathrm{~min}$, the cells were incubated with CEP for $24 \mathrm{~h}$ and then analyzed by annexin V/PI staining. In KHM-11 cells, the annexin V-positive cells induced by CEP were partly inhibited by Z-VAD-FMK, indicating that CEP induced apoptosis partly via caspase-3 activation in KHM-11 cells. However, this effect was not observed in 12PE cells.

Since ROS was induced by CEP in KHM-11 cells, the free radical scavenger, Tiron, was utilized to evaluate the role 
A

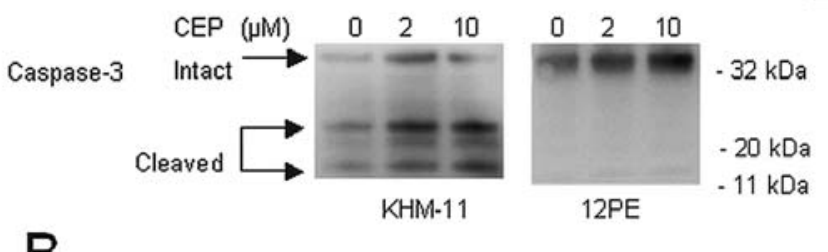

B

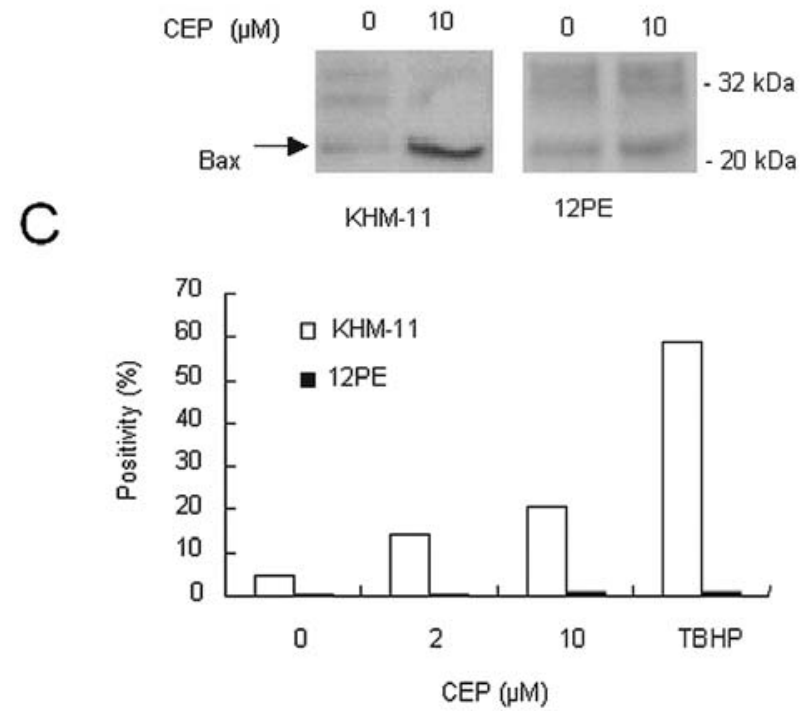

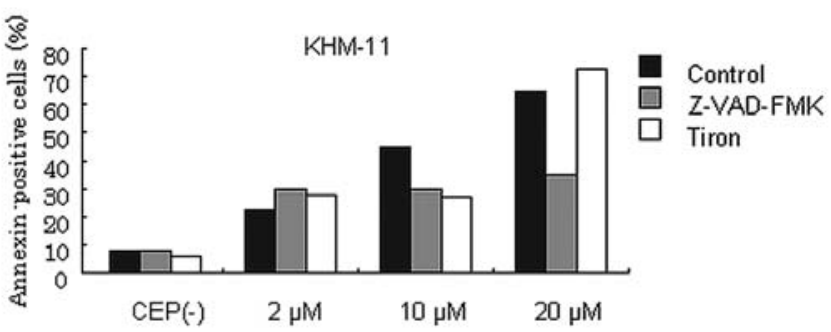

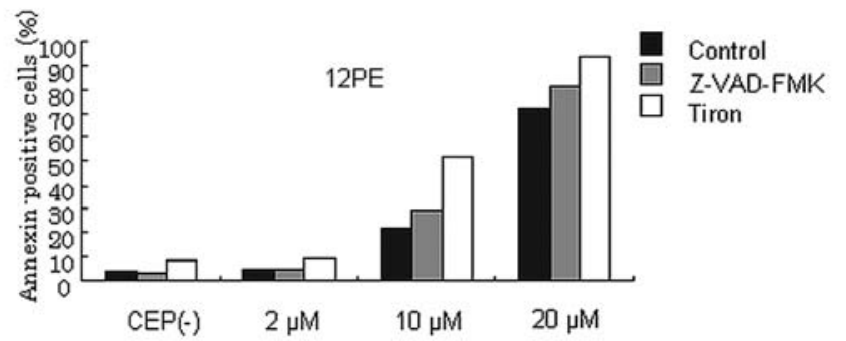

Figure 4. CEP induces activated caspase-3, Bax and ROS production. KHM-11 and 12PE cells were incubated with CEP at the indicated concentrations for $24 \mathrm{~h}$. Activation of caspase-3 (A) and induction of Bax (B) are detected in KHM-11 cells, although not in 12PE cells. (C) CEP induces the production of ROS in KHM-11 cells. KHM-11 (open bar) and 12PE (closed bar) cells were treated with CEP at the indicated concentrations for 1 h. Quantification of ROS was analyzed by flow cytometry. TBHP, t-butylhydroperoxide (positive control). CEP induces ROS in a dose-dependent manner in KHM-11 cells, though not in 12PE cells. (D) Apoptosis induced by CEP is partially inhibited by Z-VAD-FMK or Tiron in KHM-11. KHM-11 (upper panel) and 12PE cells (lower panel) were pretreated with the caspase inhibitor Z-VAD-FMK or free radical scavenger Tiron for 30 min prior to the addition of CEP. After incubation with CEP for $24 \mathrm{~h}$, the cells were subjected to annexin V/PI analysis. The percentages of annexin V-positive cells are indicated. Z-VAD-FMK and Tiron partially inhibit apoptosis induced by CEP in KHM-11 cells, though have no effect on 12PE cells.

of ROS in CEP-induced apoptosis (Fig. 4D). Cells were pretreated with Tiron, followed by treatment with CEP. Tiron partly inhibited apoptosis in KHM-11 cells exposed to CEP at a concentration of $10 \mu \mathrm{M}$, though not in 12PE cells at any concentrations of CEP. However, in the presence of $20 \mu \mathrm{M}$ CEP, Tiron did not inhibit apoptosis in KHM-11 cells, suggesting that the cell death at this high concentration of CEP may not be mediated by ROS.

Taken together, these results suggest that CEP-mediated inhibition of survival in 12PE cells is distinct from that in KHM-11 cells.

Cell cycle arrest by CEP. Since cell survival was inhibited by CEP in KHM-11 and 12PE cells, although caspase- 3 and ROS were not involved in $12 \mathrm{PE}$, we then examined the cell cycles of CEP-treated cells. As shown in Fig. 5A and B, CEP treatment resulted in dose-dependent decreases in cells at the S-phase and an increase of the G0/G1 phase in KHM-11 and $12 \mathrm{PE}$ cells. Interestingly, the reduction of cells at the $\mathrm{S}$-phase was more evident in KHM-11 cells than in $12 \mathrm{PE}$ cells. Accumulation of cells at the sub-G1 state was clearly observed in KHM-11 cells, but not in 12PE cells. As found in $12 \mathrm{PE}$ cells, U266 cells showed marked reduction of the S-phase with no increase of the sub-G1 fraction (data not shown).
$C E P$ up regulates $C D K$ inhibitors. To further elucidate the mechanisms by which CEP regulates the cell cycle, the expression levels of cyclins, cyclin-dependent kinases (CDKs; CDK-4, CDK-6) and CDK inhibitors (p15 INK4B, p16 INK4A, p21 Waf1/Cip1, p27 Kip1) as well as the phosphorylation of $\mathrm{Rb}$ were analyzed by Western blotting (Fig. 6A). In CEP-treated KHM-11 cells, the levels of cyclin D1, CDK-6, p15 INK4B and p16 INK4A were all decreased. Notably, however, an increase of p15 INK4B and p21 Waf1/Cip1 was found in CEP-treated 12PE cells while cyclin D1 and CDK-6 decreased. A decrease in phosphorylated $\mathrm{Rb}$ was also observed in CEP-treated 12PE cells.

Since KHM-11 rapidly underwent apoptosis by CEP via caspase-3, CDK inhibitors were analyzed in KHM-11 under caspase-inhibition. When caspase was inhibited by a pancaspase inhibitor, ZVAD-FMK, in KHM-11 cells, marked induction of p15 INK4B was found (Fig. 6B), indicating that rapid induction of apoptosis may precede the induction of $\mathrm{p} 15$ INK4B in KHM-11 cells. However, the expression of $21 \mathrm{Waf} /$ Cip1 was not induced at the same condition (data not shown).

\section{Discussion}

In the present study, anti-myeloma effects of CEP were identified in vitro as well as in vivo. CEP inhibited the growth 
A
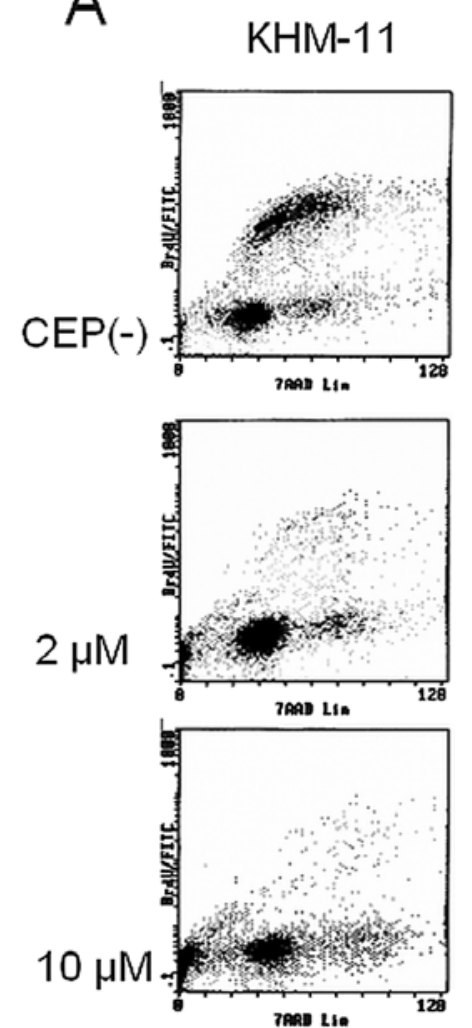

$12 \mathrm{PE}$
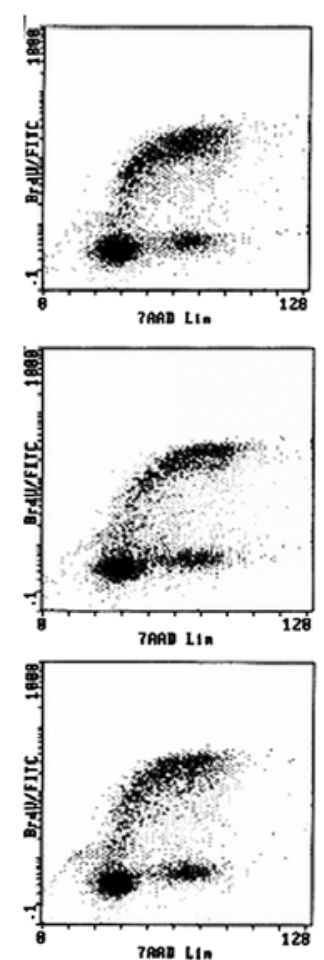

B
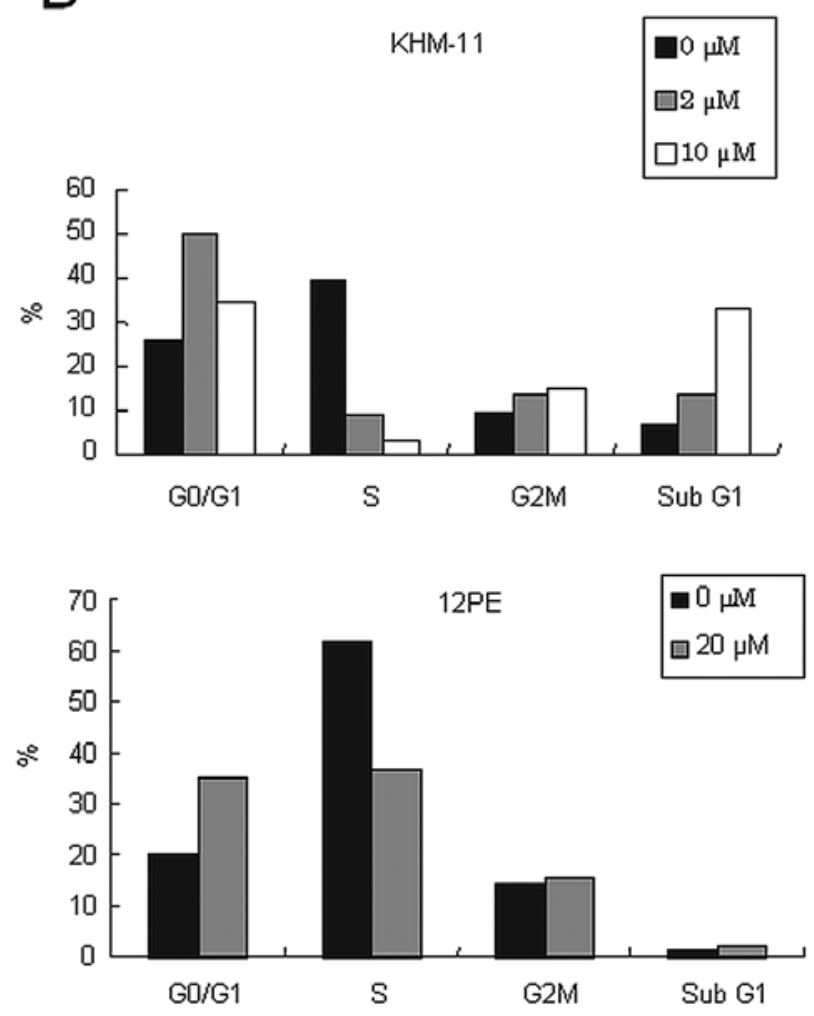

Figure 5. CEP induces a reduction in S-phase cells and an increase in G0/G1-phase cells. (A) Cells were treated with CEP at the indicated concentrations for $24 \mathrm{~h}$ and the cell cycle was analyzed by flow cytometry. (B) Quantification of the results. Upper panel, KHM-11; lower panel, 12PE. A marked decrease in S-phase cells and an increase in sub-G1 cells are observed in KHM-11 cells. Although 12PE cells also show a marked decrease in S-phase cells, there is no increase in sub-G1 cells.

A

KHM-11

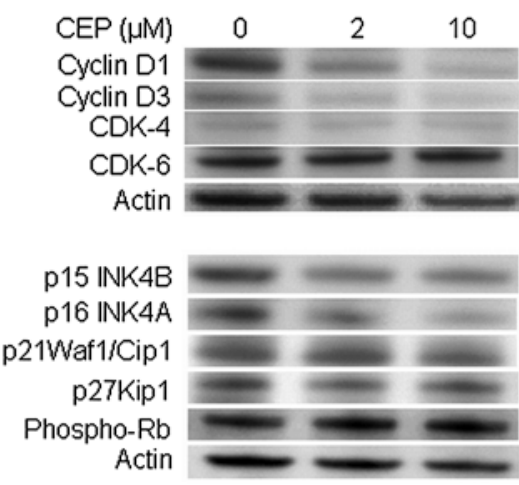

B

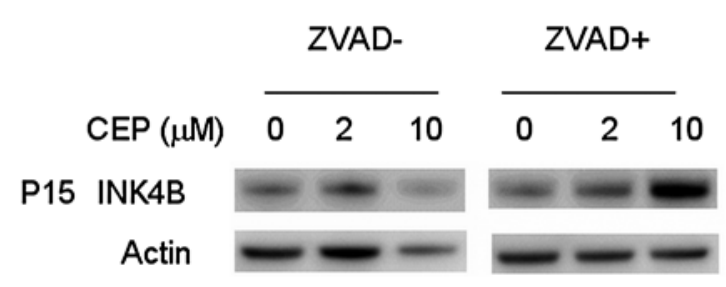

Figure 6. Induction of p15 INK4B and p21 INK4A by CEP. (A) The expression levels of cyclins, CDKs, CDK inhibitors and phosphorylated Rb were analyzed by Western blotting. The cells were treated with CEP at the indicated concentrations for $24 \mathrm{~h}$ prior to analysis. The levels of cyclin D1, p15 INK4B and p16 INK4A are decreased in a dose-dependent manner in KHM-11 cells. However, marked increases in p15 INK4B and p21 Waf1 are observed in 12PE cells, in accordance with decreases in CDK-6 and cyclin D1. (B) Expression of p15 INK4B was analyzed by Western blotting in KHM-11 under the inhibition of caspase-3. Marked induction of p15 INK4B was found by the treatment with ZVAD-FMK in KHM-11 cells. 
of all myeloma cells at concentrations ranging from 1-10 $\mu \mathrm{M}$, which are reported to be clinically achievable, thereby suggesting the efficacy of CEP in the clinical setting.

Several mechanisms for CEP-mediated inhibition of tumor cell survival have been proposed (6). It has been reported that CEP alone can directly induce apoptosis in a variety of cancer cells $(17,18,20,23,32,33)$. Production of ROS in neutrophils (34) and induction of p27 in squamous cell carcinoma by CEP (17) might be involved in a direct anti-cancer effect. CEP is also known to be an inhibitor of NFKB (7). It is noteworthy that CEP is capable of inducing sensitivity to anticancer agents and overcoming drug resistance when utilized with other chemotherapeutic agents (10,12-15,35).

Based on these scattered preceding reports and our experience of a myeloma patient showing response to CEP, we investigated the functions of CEP by Western blot analysis targeting apoptosis-related proteins, cell cycle analysis, detection of ROS production and inhibition of caspase- 3 or ROS scavenger pathways. Our results clearly show that CEP induces growth inhibition through two major mechanisms, namely the induction of apoptosis involving ROS, Bax and caspase-3, and cell cycle arrest by induction of CDK inhibitors and downregulation of CDKs. However, the manner in which these two pathways are distinctly regulated remains unknown. It is possible that CEP always tends to induce cell cycle arrest, though apoptosis may precede in cells where apoptosis is not inhibited. Indeed, when apoptosis was inhibited by a caspase inhibitor, induction of p15 INK4B was observed in KHM-11. Therefore, CEP may simultaneously induce the activation of caspases and induction of CDK inhibitors.

We observed the inhibition of survival by CEP even in melphalan-resistant cells, indicating the possibility that CEP may overcome drug resistance. Similar studies have shown sensitization to chemotherapy by CEP in chemotherapyresistant tumors $(8,36)$. These results suggest that CEP may contribute to overcoming acquired drug resistance.

As we found that CEP was not toxic at $2 \mu \mathrm{M}$, which is $\mathrm{IC}_{50}$ of CEP to KHM-11 cells, to lymphocytes from a normal volunteer with or without stimulation of LPS, respectively (data not shown), safety of CEP to normal tissues is suggested. This was also supported by our observation showing no adverse effects in our patient. Safety of CEP has been well accepted in Japan for decades since it became available.

Since CEP includes four structurally related compounds, namely CEP, berbamine, cycleanine and isotetrandrine, the growth inhibitory effects of these compounds, except for cycleanine, were analyzed by the WST- 8 assay. The antimyeloma effects of these compounds did not differ significantly, although the most potent activity was found for purified CEP (data not shown). Zhao et al (37) reported that berbamine, a structurally similar compound to CEP, inhibits survivin by binding to the survivin promoter in leukemia cells. Taking the marked structural similarity of berbamine to CEP into account, our results suggest that CEP plays an important role not only in anti-myeloma activity but also in anti-leukemia cells.

Our experiments arose from clinical observations of a myeloma case that responded to CEP. The case did not show any adverse effects and remained in a plateau phase for $>6$ months on a combination of CEP and dexamethasone.
CEP showed anti-myeloma effects when utilized as monotherapy while the response was transient. Since the combination of CEP with dexamethasone was clinically effective, there could be a synergistic effect by these reagents although the mechanism is unclear. We expect that the administration of this compound at a higher dose may be effective as monotherapy.

Future detailed analyses of the mechanisms of CEP for regulating cell cycle arrest and apoptosis should lead to the development of new therapeutic agents derived from the original structure of CEP. High structural similarity in CEP related compounds, berbamine, cycleanine and isotetrandrine, all of which show anti-myeloma effects, indicates that the existence of target molecule(s) of these compounds plays a critical role for survival of myeloma cells.

In the clinical setting, CEP has been utilized for many years and has proven to be non-toxic. Salvage or maintenance therapeutic strategies utilizing CEP could be tolerable candidates for MM, even for patients with a poor performance status.

\section{Acknowledgements}

This study was supported in part by a Grant for Research on Cancer from the Ministry of Education, Culture, Sports, Science and Technology of Japan.

\section{References}

1. Anderson KC, Hannah AL, Pazdur R and Farrell AT: A strategic framework for novel drug development in multiple myeloma. $\mathrm{Br}$ J Haematol 138: 153-159, 2007.

2. Richardson PG, Mitsiades C, Schlossman R, Munshi N and Anderson K: New drugs for myeloma. Oncologist 12: 664-689, 2007.

3. Tomita M, Fujitani K and Aoyagi Y: Synthesis of dlcepharanthine. Tetrahedron Lett 13: 1201-1206, 1967.

4. Nemoto K, Yoshida K, Nisimura M and Seki M: The effects of Cepharanthin on the recovery of hematopoietic stem cells after X-ray irradiation. Gan To Kagaku Ryoho 18: 81-84, 1991.

5. Takahashi K, Shinozuka T, Kiuchi K, et al: Cepharanthine therapy of alopecia. C R Seances Soc Biol Fil 150: 1844-1845, 1956.

6. Furusawa $\mathrm{S}$ and $\mathrm{Wu} \mathrm{J}$ : The effects of biscoclaurine alkaloid cepharanthine on mammalian cells: implications for cancer, shock, and inflammatory diseases. Life Sci 80: 1073-1079, 2007.

7. Azuma M, Aota K, Tamatani T, et al: Suppression of tumor necrosis factor alpha-induced matrix metalloproteinase 9 production in human salivary gland acinar cells by cepharanthine occurs via down-regulation of nuclear factor kappaB: a possible therapeutic agent for preventing the destruction of the acinar structure in the salivary glands of Sjogren's syndrome patients. Arthritis Rheum 46: 1585-1594, 2002.

8. Sumizawa T, Chen ZS, Chuman Y, et al: Reversal of multidrug resistance-associated protein-mediated drug resistance by the pyridine analog PAK-104P. Mol Pharmacol 51: 399-405, 1997.

9. Aogi K, Nishiyama M, Kim R, et al: Overcoming CPT-11 resistance by using a biscoclaurine alkaloid, cepharanthine, to modulate plasma trans-membrane potential. Int J Cancer 72: 295-300, 1997.

10. Kitazono M, Okumura H, Ikeda R, et al: Reversal of LRPassociated drug resistance in colon carcinoma SW-620 cells. Int J Cancer 91: 126-131, 2001.

11. Murakami K, Cox RA, Hawkins HK, et al: Cepharanthin, an alkaloid from Stephania cepharantha, inhibits increased pulmonary vascular permeability in an ovine model of sepsis. Shock 20: 46-51, 2003.

12. Ikeda R, Che XF, Yamaguchi T, et al: Cepharanthine potently enhances the sensitivity of anticancer agents in K562 cells. Cancer Sci 96: 372-376, 2005. 
13. Nakajima A, Yamamoto Y, Taura K, et al: Beneficial effect of cepharanthine on overcoming drug-resistance of hepatocellular carcinoma. Int J Oncol 24: 635-645, 2004.

14. Katsui K, Kuroda M, Wang Y, et al: Cepharanthin enhances adriamycin sensitivity by synergistically accelerating apoptosis for adriamycin-resistant osteosarcoma cell lines, $\mathrm{SaOS} 2-\mathrm{AR}$ and SaOS2 F-AR. Int J Oncol 25: 47-56, 2004.

15. Kono K, Takahashi JA, Ueba T, Mori H, Hashimoto N and Fukumoto M: Effects of combination chemotherapy with biscoclaurine-derived alkaloid (Cepharanthine) and nimustine hydrochloride on malignant glioma cell lines. J Neurooncol 56: 101-108, 2002.

16. Tamatani T, Azuma M, Motegi K, Takamaru N, Kawashima Y and Bando T: Cepharanthin-enhanced radiosensitivity through the inhibition of radiation-induced nuclear factor-kappaB activity in human oral squamous cell carcinoma cells. Int $\mathrm{J}$ Oncol 31: 761-768, 2007.

17. Harada K, Supriatno, Yamamoto S, Kawaguchi S, Yoshida H and Sato M: Cepharanthine exerts antitumor activity on oral squamous cell carcinoma cell lines by induction of p27Kip1. Anticancer Res 23: 1441-1448, 2003.

18. Wu J, Suzuki H, Akhand AA, Zhou YW, Hossain K and Nakashima I: Modes of activation of mitogen-activated protein kinases and their roles in cepharanthine-induced apoptosis in human leukemia cells. Cell Signal 14: 509-515, 2002.

19. Satoh K, Nagai F, Ono M and Aoki N: Inhibition of $\mathrm{Na}(+)$, $\mathrm{K}(+)$-ATPase by the extract of Stephania cephararantha HAYATA and bisbenzylisoquinoline alkaloid cycleanine, a major constituent. Biochem Pharmacol 66: 379-385, 2003.

20. Biswas KK, Tancharoen S, Sarker KP, Kawahara K, Hashiguchi T and Maruyama I: Cepharanthine triggers apoptosis in a human hepatocellular carcinoma cell line (HuH-7) through the activation of JNK $1 / 2$ and the downregulation of Akt. FEBS Lett 580: 703-710, 2006

21. Fujimura T, Furusawa S, Kawauchi H, Takayanagi Y and Sasaki K: Effect of cepharanthine on doxorubicin cytotoxicity in P388 murine leukemia cells in vitro and in vivo. Res Commun Chem Pathol Pharmacol 71: 235-238, 1991.

22. Morikawa K, Oseko F and Morikawa S: Inhibition of proliferation and differentiation of human B-lymphocytes by a biscoclaurine alkaloid. Int J Immunopharmacol 14: 941-949, 1992.

23. Wu J, Suzuki H, Zhou YW, et al: Cepharanthine activates caspases and induces apoptosis in Jurkat and K562 human leukemia cell lines. J Cell Biochem 82: 200-214, 2001.

24. Saito N, Takemori N, Hirai K, Onodera R, Watanabe S and Kohgo Y: High-dose biscoclaurine alkaloids together with prednisolone raise platelet counts in chronic idiopathic thrombocytopenic purpura. Am J Hematol 51: 173-174, 1996.
25. Kobayashi M, Katayama T, Ochiai S, et al: High-dose cepharanthin therapy of idiopathic thrombocytopenic purpura. Rinsho Ketsueki 33: 405-407, 1992.

26. Ohtsuki T, Yawata Y, Wada H, Sugihara T, Mori M and Namba M: Two human myeloma cell lines, amylase-producing KMS-12-PE and amylase-non-producing KMS-12-BM, were established from a patient, having the same chromosome marker, t(11;14)(q13;q32). Br J Haematol 73: 199-204, 1989.

27. Carini C, Hudspith BN and Brostoff J: Effect of prostaglandins and cyclic nucleotides on growth and immunoglobulin secretion of two IgE myeloma cell lines. Br J Cancer 43: 257-260, 1981.

28. Togawa A, Inoue N, Miyamoto K, Hyodo H and Namba M: Establishment and characterization of a human myeloma cell line (KMM-1). Int J Cancer 29: 495-500, 1982.

29. Hata H, Matsuzaki H, Sonoki T, et al: Establishment of a CD45-positive immature plasma cell line from an aggressive multiple myeloma with high serum lactate dehydrogenase. Leukemia 8: 1768-1773, 1994.

30. Hamburger AW, Kim MB and Salmon SE: The nature of cells generating human myeloma colonies in vitro. J Cell Physiol 98: 371-376, 1979.

31. Harada N, Nagasaki A, Hata H, Matsuzaki H, Matsuno F and Mitsuya H: Down-regulation of CD98 in melphalan-resistant myeloma cells with reduced drug uptake. Acta Haematol 103: 144-151, 2000.

32. Harada K, Bando T, Yoshida H and Sato M: Characteristics of antitumour activity of cepharanthin against a human adenosquamous cell carcinoma cell line. Oral Oncol 37: 643-651, 2001.

33. Furusawa S, Wu J, Fujimura T, et al: Cepharanthine inhibits proliferation of cancer cells by inducing apoptosis. Methods Find Exp Clin Pharmacol 20: 87-97, 1998.

34. Akamatsu H, Komura J, Asada Y and Niwa Y: Effects of cepharanthin on neutrophil chemotaxis, phagocytosis, and reactive oxygen species generation. J Dermatol 18: 643-648, 1991.

35. Wang Y, Kuroda M, Gao XS, et al: Cepharanthine enhances in vitro and in vivo thermosensitivity of a mouse fibrosarcoma, FSa-II, based on increased apoptosis. Int J Mol Med 13: 405-411, 2004.

36. Hotta $\mathrm{T}$, Tanimura $\mathrm{H}$, Yamaue $\mathrm{H}$, et al: Modulation of multidrug resistance by cepharanthine in fresh human gastrointestinal tumor cells. Oncology 54: 153-157, 1997.

37. Zhao XY, He ZW, Wu D and Xu RZ: Berbamine selectively induces apoptosis of human acute promyelocytic leukemia cells via survivin-mediated pathway. Chin Med J (Engl) 120: 802-806, 2007. 